\title{
THE PROPER-TIME LORENTZ GROUP DEMYSTIFIED
}

\author{
ABRAHAM A. UNGAR
}

Communicated by Izu Vaisman

\begin{abstract}
Proper velocities are measured by proper time as opposed to coordinate velocities, which are measured by coordinate time. The standard Lorentz transformation group, in which each transformation is expressed by a coordinate velocity and an orientation between two inertial frames, is well known. In contrast, the equivalent proper-time Lorentz transformation group, in which each transformation is expressed by a proper velocity and an orientation between two inertial frames is unknown. The dignity of special relativity theory requires that every possible means be explored for the solution of a problem so elegant and so celebrated. Fortunately, a so called gyro-formalism approach to special relativity enables the elusive proper-time Lorentz transformation group to be uncovered.
\end{abstract}

\section{Introduction}

Einstein velocity addition is a century-old idea whose time has come back. Following the discovery of the gyrovector space structure to which Einstein velocity addition gives rise [26], and unleashing the power of its hyperbolic geometry [27], hitherto important unsolved problems of special relativity theory can be solved. The problem in case is the determination of the proper-time Lorentz transformation group, a problem that eluded all its previous explorers as we demonstrate in this article.

Coordinate time, or observer's time, is the time $t$ of a moving object measured by an observer at rest. Accordingly, special relativity theory is formulated in terms of Coordinate time. Contrasting coordinate time, proper time, or traveller's time, is the time $\tau$ of a moving object measured by a co-moving observer. Proper time is useful, for instance, in the understanding of the twin paradox [11], and the mean life time of unstable moving particles, like muons.

The mean lifetime of muons between creation, in the upper atmosphere, and disintegration is $2.2 \mu \mathrm{s}$ (proper lifetime) measured by their proper time. This proper time of the moving muon, measured by the muon own clock, is several orders 
of magnitude shorter than the time the muon is seen travelling through the atmosphere by Earth observers. Of course, there is no need to attach a co-moving observer to the moving muon. Observers at rest measure the coordinate mean lifetime of the moving muon that, owing to time dilation, is observer dependent. Each observer, however, can translate his measure of the muon coordinate mean lifetime into the muon proper mean lifetime, which is an intrinsic property of the muon and hence observer independent [6].

The need to reformulate relativity physics in terms of proper time instead of coordinate time arises from time to time - see, for instance, references $[10,16,32]$.

As a result, derivations of various proper-time Lorentz transformations are available in the literature. Unfortunately, non of the resulting "proper-time Lorentz transformation groups" is equivalent to the standard, coordinate-time Lorentz transformation group.

Since 1993 Gill, Lindesay, and Zachary (GLZ) have been emphasizing the need by developing a proper time formulation and studying its consequences in order to gain new insights, see [15] and references therein. Their reformulation of relativity physics is based on the convention of replacing coordinate time by proper time and, perhaps, other conventions. The convention(s) led them to a new invariance group that they describe in [9] as a "new symmetry group which is distinct from, but closely related to the Lorentz group".

Gill, Lindesay, and Zachary agree that the passage from coordinate time to proper time is a matter of convention. They, however, claim that this convention leads to new physical matters of experimental fact. They thus write:

The above transformations [of the proper-time group] are so close to Lorentz transformations that one might wander if any new physics is possible. Not only is there new physics, as we will be seen later, but ...

GLZ [9, p. 1314]

and

The work of this paper shows that different conventions can lead to different physical theories.

GLZ [9, p. 1349].

Physical theories are different if, and only if, they are experimentally distinguishable. Indeed, Gill, Lindesay, and Zachary report that their proper-time considerations give rise to a new result that verifies that their proper-time relativistic theory is experimentally distinguishable from Einstein's special relativity: 
In this paper we show that Maxwell's equations have a mathematically equivalent formulation [proper-time Maxwell equations]. However, the speed of light now depends on the motion of the source.

GLZ [9, p. 1347].

In contrast, in Einstein's special relativity the speed of light is source independent. It is a universal constant $c$, where $c<\infty$ if measured by coordinate time, and $c=\infty$ if measured by proper time.

The independence of the speed of light in vacuum on the speed of its source is a matter of experimental fact. Thus, the claim of Gill, Lindesay, and Zachary that different conventions can lead to different physical theories amounts to a claim that matters of convention can give rise to matters of experimental fact. The absurdity in extracting matters of experimental fact from matters of convention indicates that the way in which Gill, Lindesay, and Zachary have implemented the convention of replacing the standard, coordinate-time Lorentz transformation group by a corresponding proper-time Lorentz transformation group must be erroneous. Of course, one may argue that Gill, Lindesay, and Zachary did not obtain new physics directly from the convention of replacing coordinate time by proper time. Rather, they have exploited the convention merely to extend the validity of special relativity by another convention, and it is the extended relativity that gives rise to their new physics. However, our point is that Gill, Lindesay, and Zachary did not obtain a proper-time Lorentz group that is equivalent to the standard Lorentz group, as it should. In contrast, we present a method of uniquely constructing the proper-time Lorentz group, which is demystified by a demonstration that it is equivalent to the standard Lorentz group algebraically, geometrically and, hence, experimentally.

In [8] Gill and Zachary inform that their proper time formulation is related to the work of Wegener [30], who showed that the use of the proper time allows the construction of Galilean transformations from Lorentz transformations. Indeed, Wegener claims in [30] that "proper time being invariant, the transformations of coordinates must be Galilean". Wegener, accordingly, proposes "a classical alternative to special relativity" which is experimentally slightly different from Einstein's special relativity. Thus, the convention of replacing coordinate time by proper time results in new physical insights, different from those of special relativity.

Accordingly, the aim of this article is to uncover the unique way (in some specified sense) to translate the standard, coordinate-time Lorentz transformation group into a corresponding proper-time Lorentz transformation group. To perform the 
translation we must first understand the algebraic "gyrostructure" of the standard (homogeneous, proper, orthochronous) Lorentz group in terms of Einstein velocity addition. The passage to the proper-time Lorentz transformation group will then become natural and unique, leaving no room for ambiguities. The gyrostructure of the standard Lorentz group that we employ to uncover the proper-time Lorentz transformation group is presented in [26]. Therefore, the proof of some statements will be omitted, referring interested readers to [26]. As expected, the resulting proper-time Lorentz group is experimentally indistinguishable from its standard, coordinate-time counterpart.

The task of understanding the actual algebraic structure of the standard Lorentz group is culminated in Section 6 with the presentation of the abstract Lorentz group. To improve our understanding of the abstract Lorentz group we present in Sections 7, 9, 10 and 11 four examples that illustrate the realization of the abstract Lorentz group by various concrete Lorentz groups. One of the four examples, in Section 9, presents the proper-time Lorentz group, thus completing the main task of this article.

\section{The Abstract Spacetime Event}

Let $\mathbb{R}$ be the real line and let $\mathbb{V}$ be the abstract real inner product space. Furthermore, let

$$
\mathbb{V}_{c}=\{\mathbf{v} \in \mathbb{V} ;\|\mathbf{v}\|<c\}
$$

be the ball of radius $c$ of $\mathbb{V}$, where $c>0$ is an arbitrarily fixed positive constant representing the vacuum speed of light.

The pair

$$
\left(\begin{array}{c}
t \\
\mathbf{v} t
\end{array}\right)=\left(\begin{array}{c}
t \\
\mathbf{x}
\end{array}\right)
$$

$t \in \mathbb{R}, \mathbf{v} \in \mathbb{V}_{c}, \mathbf{x} \in \mathbb{V}$, is called a spacetime event, where $t$ denotes time, $\mathbf{x}$ denotes space, and $\mathbf{v}$ denotes relativistically admissible velocity.

The symbol " $t$ " that appears on the left hand side of (2) two times must sometimes be substituted by involved expressions. Hence, without loss of information, and for convenience, we identify the pair in (2) with the pair

$$
\left(\begin{array}{c}
t \\
\mathbf{v}
\end{array}\right)
$$

in which the symbol " $t$ " appears once rather than twice. Accordingly, we call (2) and (3), respectively, the space notation and the velocity notation of spacetime. 
Clearly, the space notation and the velocity notation are equivalent for all $t>0$ (and for all $t<0$ as well). We will use the velocity notation mainly in the manipulation of intermediary results. Once a final result is obtained, we will register it in the customary space notation.

Recalling that a nonempty set with a binary operation is called a groupoid, we introduce a binary operation $\oplus_{\mathrm{E}}$ into the ball $\mathbb{V}_{c}$, turning it into a groupoid. The binary operation is given by the equation

$$
\mathbf{u} \oplus_{\mathrm{E}} \mathbf{v}=\frac{1}{1+\frac{\mathbf{u} \cdot \mathbf{v}}{c^{2}}}\left\{\mathbf{u}+\frac{1}{\gamma_{\mathbf{u}}} \mathbf{v}+\frac{1}{c^{2}} \frac{\gamma_{\mathbf{u}}}{1+\gamma_{\mathbf{u}}}(\mathbf{u} \cdot \mathbf{v}) \mathbf{u}\right\}
$$

for all $\mathbf{u}, \mathbf{v} \in \mathbb{V}_{c}$, where $\cdot$ and $\|\cdot\|$ are the inner product and norm that the ball $\mathbb{V}_{c}$ inherits from its space $\mathbb{V}$, and where $\gamma_{\mathbf{u}}$ is the Lorentz gamma factor

$$
\gamma_{\mathbf{v}}=\frac{1}{\sqrt{1-\frac{\|\mathbf{v}\|^{2}}{c^{2}}}}
$$

The binary operation $\bigoplus_{\mathrm{E}}$ is recognized as Einstein addition of relativistically admissible velocities [2,3,20], satisfying the gamma identity [27]

$$
\gamma_{\mathbf{u} \oplus_{\mathrm{E}} \mathbf{v}}=\gamma_{\mathbf{u}} \gamma_{\mathbf{v}}\left(1+\frac{\mathbf{u} \cdot \mathbf{v}}{c^{2}}\right)
$$

or equivalently

$$
\frac{\gamma_{\mathbf{u} \oplus_{\mathrm{E}} \mathbf{v}}}{\gamma_{\mathbf{u}} \gamma_{\mathbf{v}}}=1+\frac{\mathbf{u} \cdot \mathbf{v}}{c^{2}}, \quad \frac{\gamma_{\mathbf{u} \ominus_{\mathrm{E}} \mathbf{v}}}{\gamma_{\mathbf{u}} \gamma_{\mathbf{v}}}=1-\frac{\mathbf{u} \cdot \mathbf{v}}{c^{2}}
$$

for all $\mathbf{u}, \mathbf{v} \in \mathbb{V}_{c}$. Einstein subtraction, denoted by $\ominus_{\mathrm{E}}$ is given by the equation $\mathbf{u} \ominus_{\mathrm{E}} \mathbf{v}=\mathbf{u} \oplus_{\mathrm{E}}(-\mathbf{v})$.

For applications in special relativity one realizes the abstract real inner product space $\mathbb{V}$ by the Euclidean 3 -space $\mathbb{R}^{3}$, so that Einstein velocity addition of special relativity, given by (4), becomes a binary operation in the Euclidean 3-dimensional ball $\mathbb{R}_{c}^{3}$

$$
\mathbb{R}_{c}^{3}=\left\{\mathbf{v} \in \mathbb{R}^{3} ;\|\mathbf{v}\|<c\right\}
$$

\section{The Einstein Velocity Gyrogroup}

Being neither commutative nor associative, Einstein addition $\bigoplus_{\mathrm{E}}$ is seemingly structureless. It is therefore interesting to realize that Einstein groupoid $\left(\mathbb{V}_{c}, \oplus_{\mathrm{E}}\right)$ 
has a grouplike structure called a gyrocommutative gyrogroup, a term that was coined in [24]. Accordingly, an Einstein groupoid is called an Einstein gyrogroup. Being a gyrocommutative gyrogroup operation, Einstein addition $\bigoplus_{\mathrm{E}}$ is gyrocommutative and gyroassociative just as a commutative group operation (like vector addition) is commutative and associative.

To present the gyrocommutative and the gyroassociative laws of Einstein addition we define the gyro-operation

$$
\text { gyr : } \mathbb{V}_{c} \times \mathbb{V}_{c} \rightarrow \operatorname{Aut}\left(\mathbb{V}_{c}, \oplus_{\mathrm{E}}\right)
$$

which generates gyrations gyr $[\mathbf{u}, \mathbf{v}]: \mathbb{V}_{c} \rightarrow \mathbb{V}_{c}, \mathbf{u}, \mathbf{v} \in \mathbb{V}_{c}$, according to the equation

$$
\operatorname{gyr}[\mathbf{u}, \mathbf{v}] \mathbf{w}=\ominus_{\mathrm{E}}\left(\mathbf{u} \oplus_{\mathrm{E}} \mathbf{v}\right) \oplus_{\mathrm{E}}\left\{\mathbf{u} \oplus_{\mathrm{E}}\left(\mathbf{v} \oplus_{\mathrm{E}} \mathbf{w}\right)\right\}
$$

for all $\mathbf{u}, \mathbf{v}, \mathbf{w} \in \mathbb{V}_{c}$. Clearly, Einstein gyrations (10) measure nonassociativity in Einstein addition. In the special case of parallel velocities, when Einstein addition is associative, Einstein gyrations are indeed trivial (a trivial map being the identity map I). Thus, if $\mathbf{u}$ and $\mathbf{v}$ are parallel, then gyr $[\mathbf{u}, \mathbf{v}]=\mathrm{I}$.

Einstein addition possesses the left cancellation law

$$
\ominus_{\mathrm{E}} \mathbf{u} \oplus_{\mathrm{E}}\left(\mathbf{u} \oplus_{\mathrm{E}} \mathbf{v}\right)=\mathbf{v}
$$

for all $\mathbf{u}, \mathbf{v} \in \mathbb{V}_{c}$ (but, surprisingly, it does not possess a similar right cancellation law, see [26]). Hence, for instance

$$
\operatorname{gyr}[\mathbf{0}, \mathbf{v}] \mathbf{w}=\mathbf{w}, \quad \operatorname{gyr}[\mathbf{v}, \mathbf{0}] \mathbf{w}=\mathbf{w}
$$

so that both gyr $[\mathbf{0}, \mathbf{v}]$ and gyr $[\mathbf{v}, \mathbf{0}]$ are trivial for all $\mathbf{v} \in \mathbb{V}_{c}$, being the identity automorphisms of the groupoid $\left(\mathbb{V}_{c}, \oplus_{\mathrm{E}}\right)$.

We recall that an automorphism of a groupoid $(G, \oplus)$ is a bijective self-map of $G$ that preserves the binary operation $\oplus$ in $G$. The set of all automorphisms of a groupoid $(G, \oplus)$ forms a group denoted $\operatorname{Aut}(G, \oplus)$. It is shown in [26] that the gyrations gyr $[\mathbf{u}, \mathbf{v}]$ of $\mathbb{V}_{c}$ are automorphisms of the groupoid $\left(\mathbb{V}_{c}, \oplus_{\mathrm{E}}\right)$ for any $\mathbf{u}, \mathbf{v} \in \mathbb{V}_{c}$

$$
\operatorname{gyr}[\mathbf{u}, \mathbf{v}] \in \operatorname{Aut}\left(\mathbb{V}_{c}, \oplus_{\mathrm{E}}\right) .
$$

Hence, the gyrations gyr $[\mathbf{u}, \mathbf{v}], \mathbf{u}, \mathbf{v} \in \mathbb{V}_{c}$, are also called gyroautomorphisms. It can be shown that there are automorphisms in $\operatorname{Aut}\left(\mathbb{V}_{c}, \oplus_{\mathrm{E}}\right)$ that are not gyroautomorphisms [26], and that the inverse of a gyroautomorphism is, again, a gyroautomorphism

$$
(\operatorname{gyr}[\mathbf{u}, \mathbf{v}])^{-1}=\operatorname{gyr}[\mathbf{v}, \mathbf{u}] .
$$


In general, the composition of two gyroautomorphisms need not be a gyroautomorphism.

The gyroautomorphisms gyr $[\mathbf{u}, \mathbf{v}], \mathbf{u}, \mathbf{v} \in \mathbb{V}_{c}$, regulate Einstein addition, forcing it to be gyrocommutative and gyroassociative. As such, they form the missing link between Einstein addition and ordinary vector addition. They give rise to the gyrocommutative law of Einstein addition

$$
\mathbf{u} \oplus_{\mathrm{E}} \mathbf{v}=\operatorname{gyr}[\mathbf{u}, \mathbf{v}]\left(\mathbf{v} \oplus_{\mathrm{E}} \mathbf{u}\right)
$$

and to the gyroassociative law (left and right) of Einstein addition

$$
\begin{aligned}
& \mathbf{u} \oplus_{\mathrm{E}}\left(\mathbf{v} \oplus_{\mathrm{E}} \mathbf{w}\right)=\left(\mathbf{u} \oplus_{\mathrm{E}} \mathbf{v}\right) \oplus_{\mathrm{E}} \operatorname{gyr}[\mathbf{u}, \mathbf{v}] \mathbf{w} \\
& \left(\mathbf{u} \oplus_{\mathrm{E}} \mathbf{v}\right) \oplus_{\mathrm{E}} \mathbf{w}=\mathbf{u} \oplus_{\mathrm{E}}\left(\mathbf{v} \oplus_{\mathrm{E}} \operatorname{gyr}[\mathbf{v}, \mathbf{u}] \mathbf{w}\right) .
\end{aligned}
$$

Furthermore, they possess a rich structure, including the loop property (left and right)

$$
\operatorname{gyr}[\mathbf{u}, \mathbf{v}]=\operatorname{gyr}\left[\mathbf{u} \oplus_{\mathrm{E}} \mathbf{v}, \mathbf{v}\right], \quad \operatorname{gyr}[\mathbf{u}, \mathbf{v}]=\operatorname{gyr}\left[\mathbf{u}, \mathbf{v} \oplus_{\mathrm{E}} \mathbf{u}\right]
$$

The gyration in the gyrocommutative law takes an Einstein sum $\mathbf{v} \oplus_{\mathrm{E}} \mathbf{u}$ into the reversely ordered Einstein sum $\mathbf{u} \oplus_{\mathrm{E}} \mathbf{v}$. As such, this effect of the gyration is recognized as the familiar effect of Thomas precession, which was already known to Silberstein in 1914 [21]. Hence, the gyroautomorphism notion extends the Thomas precession effect by abstraction. Accordingly, the abstract Thomas precessions are the Thomas gyrations from which our gyrolangauge stems.

While the discovery that the presence of Thomas precession "repairs" the breakdown of commutativity in Einstein velocity addition dates back to the infancy of special relativity, it has gone unnoticed that the presence of Thomas precession "repairs" the breakdown of associativity in Einstein velocity addition as well. The discovery of the gyroassociative law of Einstein addition in 1988 [22], prompted i) the development of the so called K-loop Theory [13], a term coined by the author in 1989 [23], and ii) the development of the theory of gyrogroups and gyrovector spaces, terms coined by the author later [24-26]. The history of gyrogroup theory and its emergence in relativity physics is presented in [20, pp. 141-142].

Einstein addition $\oplus_{\mathrm{E}}$ thus gives rise to a concrete example of a gyrocommutative gyrogroup $\left(\mathbb{V}_{c}, \oplus_{\mathrm{E}}\right)$. Gyrogroups $(G, \oplus)$, both gyrocommutative and nongyrocommutative, abound in group theory as evidenced from [4,5]. The theory of gyrogroups, developed in [26], shares remarkable analogies with group theory.

Modelled on Einstein addition and guided by analogies with groups, the definition of gyrogroups follows. 
Definition 1 (Gyrogroups) A groupoid $(G, \oplus)$ is a gyrogroup if its binary operation satisfies the following axioms. In $G$ there is at least one element, 0 , called a left identity, satisfying

G1)

$$
0 \oplus a=a
$$

for all $a \in G$. There is an element $0 \in G$ satisfying axiom $\mathrm{G} 1)$ such that for each $a \in G$ there is an element $\ominus a \in G$, called a left inverse of a, satisfying

$$
\ominus a \oplus a=0 .
$$

Moreover, for any $a, b, c \in G$ there exists a unique element gyr $[a, b] c \in G$ such that the binary operation obeys the left gyroassociative law

$$
a \oplus(b \oplus c)=(a \oplus b) \oplus \operatorname{gyr}[a, b] c .
$$

The map $\operatorname{gyr}[a, b]: G \rightarrow G$ given by $c \mapsto \operatorname{gyr}[a, b] c$ is an automorphism of the groupoid $(G, \oplus)$

$$
\operatorname{gyr}[a, b] \in \operatorname{Aut}(G, \oplus)
$$

and the automorphism gyr $[a, b]$ of $G$ is called the gyroautomorphism of $G$ generated by $a, b \in G$. The operation gyr : $G \times G \rightarrow \operatorname{Aut}(G, \oplus)$ is called the gyrooperation of $G$. Finally, the gyroautomorphism gyr $[a, b]$ generated by any $a, b \in G$ possesses the left loop property

$$
\operatorname{gyr}[a, b]=\operatorname{gyr}[a \oplus b, b] \text {. }
$$

The gyrogroup axioms in Definition 1 are classified into three classes.

1) The first pair of axioms, G1) and G2), is a reminiscent of the group axioms;

2) The last pair of axioms, G4) and G5), presents the gyrooperation axioms, and

3) The middle axioms, G3), is a hybrid axiom linking the two pairs of axioms in 1) and 2).

As in group theory, we use the notation

$$
a \ominus b=a \oplus(\ominus b)
$$

in gyrogroup theory as well.

In full analogy with groups, gyrogroups are classified into gyrocommutative and non-gyrocommutative gyrogroups.

Definition 2 (Gyrocommutative Gyrogroups) A gyrogroup $(G, \oplus)$ is gyrocommutative if its binary operation obeys the gyrocommutative law 
G6)

$$
a \oplus b=\operatorname{gyr}[a, b](b \oplus a)
$$

for all $a, b \in G$.

Einstein's failure to recognize and advance the gyrocommutative gyrogroup structure that underlies his relativistic velocity addition law contributed to the eclipse of his velocity addition of relativistically admissible 3 -velocities, creating a void that could be filled only with the Lorentz transformation of 4-velocities. In the gyro-approach to special relativity the Lorentz transformation of 4-vectors is no longer considered as a primitive notion but, rather, as a notion derived from Einstein addition, as we will see in the sequel.

\section{The Spacetime Gyrogroup}

We now wish to extend the gyrocommutative gyrogroup $(G, \oplus)$ to the larger gyrocommutative gyrogroup of pairs $(t, \mathbf{v})^{t}, t \in \mathbb{R}, \mathbf{v} \in G$, where the exponent $t$ denotes transposition. If one realizes the abstract gyrocommutative gyrogroup $(G, \oplus)$ by the concrete example of Einstein gyrogroup $\left(\mathbb{V}_{c}, \oplus_{\mathrm{E}}\right)$ then its extended gyrogroup of pairs $(t, \mathbf{v})^{t}, t \in \mathbb{R}, \mathbf{v} \in \mathbb{V}_{c}$ will naturally be viewed as a gyrogroup of spacetime events, where events are registered in their velocity notation (3).

Let $\rho: G \rightarrow \mathbb{R}^{>0}=\{r \in \mathbb{R} ; r>0\}$ be any given positive valued function defined on the gyrocommutative gyrogroup $(G, \oplus)$, which is invariant under the gyroautomorphisms of $G$

$$
\rho(\mathbf{w})=\rho(\operatorname{gyr}[\mathbf{u}, \mathbf{v}] \mathbf{w})
$$

for all $\mathbf{u}, \mathbf{v}, \mathbf{w} \in G$, and which is normalized by the condition

$$
\rho(\mathbf{0})=1 \text {. }
$$

The map $\rho$ is called a cocyclic map of the spacetime gyrocommutative gyrogroup $(\mathbb{R} \times G, \oplus, \cdot, \rho)$, which is described below.

The product of two spacetime events $(s, \mathbf{u})^{t}$ and $(t, \mathbf{v})^{t}$ in the gyrocommutative gyrogroup spacetime $(\mathbb{R} \times G, \oplus, \cdot, \rho)$ is given by the equation

$$
\left(\begin{array}{l}
s \\
\mathbf{u}
\end{array}\right) \cdot\left(\begin{array}{c}
t \\
\mathbf{v}
\end{array}\right)=\left(\begin{array}{c}
\frac{\rho(\mathbf{u} \oplus \mathbf{v})}{\rho(\mathbf{u}) \rho(\mathbf{v})} s t \\
\mathbf{u} \oplus \mathbf{v}
\end{array}\right) .
$$

The groupoid consisting of all spacetime events in $(\mathbb{R} \times G, \oplus, \cdot, \rho)$, with the binary operation given by (21), forms a gyrocommutative gyrogroup. This technique 
of extending the gyrocommutative gyrogroup $(G, \oplus)$ into the gyrocommutative gyrogroup $(\mathbb{R} \times G, \oplus, \cdot \rho)$ is analogous to a well known technique of commutative group extension in group theory [19].

The inner product, $\left\langle(s, \mathbf{u})^{t},(t, \mathbf{v})^{t}\right\rangle$, of two spacetime events, written in the velocity notation (3), is a nonnegative number given by the equation

$$
\left\langle\left(\begin{array}{l}
s \\
\mathbf{u}
\end{array}\right),\left(\begin{array}{c}
t \\
\mathbf{v}
\end{array}\right)\right\rangle=\frac{\rho(\mathbf{u} \ominus \mathbf{v})}{\rho(\mathbf{u}) \rho(\mathbf{v})} s t .
$$

The seemingly non-intuitive expressions on the right hand sides of (21) and (22), which involve the cocyclic map $\rho(\mathbf{v})$ with various arguments, are nothing else but the abstraction of (7).

The squared norm of any spacetime event $(t, v)^{t}$ is accordingly

$$
\left\|\left(\begin{array}{l}
t \\
\mathbf{v}
\end{array}\right)\right\|^{2}=\left\langle\left(\begin{array}{l}
t \\
\mathbf{v}
\end{array}\right),\left(\begin{array}{l}
t \\
\mathbf{v}
\end{array}\right)\right\rangle
$$

so that, by (22) and (20)

$$
\left\|\left(\begin{array}{l}
t \\
\mathbf{v}
\end{array}\right)\right\|=\frac{t}{\rho(\mathbf{v})}
$$

for all $\mathbf{v} \in \mathbb{V}_{c}$ and $t>0$.

\section{The Abstract Lorentz Boost}

It follows from (24) that the spacetime events $(\rho(\mathbf{v}), \mathbf{v})^{t}, \mathbf{v} \in G$, are unimodular

$$
\left\|\left(\begin{array}{c}
\rho(\mathbf{v}) \\
\mathbf{v}
\end{array}\right)\right\|=1
$$

A boost is a unimodular spacetime event. The boost parameterized by $\mathbf{v} \in G$ is thus

$$
B(\mathbf{v})=\left(\begin{array}{c}
\rho(\mathbf{v}) \\
\mathbf{v}
\end{array}\right)
$$

so that the spacetime product (21) of two boosts is a boost given by parameter gyroaddition in the gyrocommutative gyrogroup $(G, \oplus)$

$$
B(\mathbf{u}) \cdot B(\mathbf{v})=\left(\begin{array}{c}
\rho(\mathbf{u}) \\
\mathbf{u}
\end{array}\right) \cdot\left(\begin{array}{c}
\rho(\mathbf{v}) \\
\mathbf{v}
\end{array}\right)=\left(\begin{array}{c}
\rho(\mathbf{u} \oplus \mathbf{v}) \\
\mathbf{u} \oplus \mathbf{v}
\end{array}\right)=B(\mathbf{u} \oplus \mathbf{v})
$$

as one can see from (21). 
The application of the boost $B(\mathbf{u})$ to a spacetime event $(t, \mathbf{v})^{t}$ is given by spacetime product (21)

$$
B(\mathbf{u})\left(\begin{array}{c}
t \\
\mathbf{v}
\end{array}\right)=\left(\begin{array}{c}
\rho(\mathbf{u}) \\
\mathbf{u}
\end{array}\right) \cdot\left(\begin{array}{c}
t \\
\mathbf{v}
\end{array}\right)=\left(\begin{array}{c}
\frac{\rho(\mathbf{u} \oplus \mathbf{v})}{\rho(\mathbf{v})} t \\
\mathbf{u} \oplus \mathbf{v}
\end{array}\right) .
$$

Boosts preserve the inner product, that is

$$
\left\langle B(\mathbf{a})\left(\begin{array}{c}
s \\
\mathbf{u}
\end{array}\right), B(\mathbf{b})\left(\begin{array}{c}
t \\
\mathbf{v}
\end{array}\right)\right\rangle=\left\langle\left(\begin{array}{l}
s \\
\mathbf{u}
\end{array}\right),\left(\begin{array}{l}
t \\
\mathbf{v}
\end{array}\right)\right\rangle=\frac{\rho(\mathbf{u} \ominus \mathbf{v})}{\rho(\mathbf{u}) \rho(\mathbf{v})} s t
$$

for all $\mathbf{a}, \mathbf{b}, \mathbf{u}, \mathbf{v} \in \mathbb{V}_{c}$ and $s, t \in \mathbb{R}$, as one can readily check, see ([26], Theorem 10.5 , p. 316). Hence, boosts preserve the norm as well

$$
\left\|B(\mathbf{u})\left(\begin{array}{c}
t \\
\mathbf{v}
\end{array}\right)\right\|=\left\|\left(\begin{array}{c}
t \\
\mathbf{v}
\end{array}\right)\right\|=\frac{t}{\rho(\mathbf{v})}
$$

The boost product in (27) is a gyrocommutative gyrogroup operation. It is not a group operation since the set of all boosts forms a nongroup gyrogroup. While the spacetime product of two boosts is a boost, the successive application of two boosts is not equivalent to the application of a single boost. Indeed, two successive boost applications is equivalent to the application of a single boost preceded, or followed, by a gyration, as the following chain of equations demonstrates

$$
\begin{aligned}
B(\mathbf{u}) B(\mathbf{v})\left(\begin{array}{c}
t \\
\mathbf{w}
\end{array}\right) & =\left(\begin{array}{c}
\rho(\mathbf{u}) \\
\mathbf{u}
\end{array}\right) \cdot\left\{\left(\begin{array}{c}
\rho(\mathbf{v}) \\
\mathbf{v}
\end{array}\right) \cdot\left(\begin{array}{c}
t \\
\mathbf{w}
\end{array}\right)\right\}=\left(\begin{array}{c}
\rho(\mathbf{u}) \\
\mathbf{u}
\end{array}\right) \cdot\left(\begin{array}{c}
\frac{\rho(\mathbf{v} \oplus \mathbf{w})}{\rho(\mathbf{w})} t \\
\mathbf{v} \oplus \mathbf{w}
\end{array}\right) \\
& =\left(\begin{array}{c}
\frac{\rho(\mathbf{u} \oplus(\mathbf{v} \oplus \mathbf{w}))}{\rho(\mathbf{w})} t \\
\mathbf{u} \oplus(\mathbf{v} \oplus \mathbf{w})
\end{array}\right)=\left(\begin{array}{c}
\frac{\rho((\mathbf{u} \oplus \mathbf{v}) \oplus \operatorname{gyr}[\mathbf{u}, \mathbf{v}] \mathbf{w}))}{\rho(\operatorname{gyr}[\mathbf{u}, \mathbf{v}] \mathbf{w})} t \\
(\mathbf{u} \oplus \mathbf{v}) \oplus \operatorname{gyr}[\mathbf{u}, \mathbf{v}] \mathbf{w}
\end{array}\right) \\
& =\left(\begin{array}{c}
\rho(\mathbf{u} \oplus \mathbf{v}) \\
\mathbf{u} \oplus \mathbf{v}
\end{array}\right) \cdot\left(\begin{array}{c}
t \\
\operatorname{gyr}[\mathbf{u}, \mathbf{v}] \mathbf{w}
\end{array}\right)=B(\mathbf{u} \oplus \mathbf{v})\left(\begin{array}{c}
t \\
\operatorname{gyr}[\mathbf{u}, \mathbf{v}] \mathbf{w}
\end{array}\right)
\end{aligned}
$$

In the chain of equations (31) we employ the product (21), the gyroassociative law of the gyrogroup operation $\oplus,(16)$, and the invariance of the cocyclic map $\rho(\mathbf{w})$ under gyrations (19).

Suggestively, we use the notation

$$
\operatorname{Gyr}[\mathbf{u}, \mathbf{v}]\left(\begin{array}{c}
t \\
\mathbf{w}
\end{array}\right)=\left(\begin{array}{c}
t \\
\operatorname{gyr}[\mathbf{u}, \mathbf{v}] \mathbf{w}
\end{array}\right)
$$


so that the result of the chain of equations (31) can be written as the identity

$$
B(\mathbf{u}) B(\mathbf{v})\left(\begin{array}{c}
t \\
\mathbf{w}
\end{array}\right)=B(\mathbf{u} \oplus \mathbf{v}) \operatorname{Gyr}[\mathbf{u}, \mathbf{v}]\left(\begin{array}{c}
t \\
\mathbf{w}
\end{array}\right)
$$

By similar gyrogroup theoretic techniques one can also establish an identity slightly different from (33)

$$
B(\mathbf{u}) B(\mathbf{v})\left(\begin{array}{c}
t \\
\mathbf{w}
\end{array}\right)=\operatorname{Gyr}[\mathbf{u}, \mathbf{v}] B(\mathbf{v} \oplus \mathbf{u})\left(\begin{array}{c}
t \\
\mathbf{w}
\end{array}\right) .
$$

Hence, two successive boosts are equivalent to a single boost preceded (33), or followed (34), by a Thomas gyration.

Contrasting the general application of successive boosts, which involves Thomas gyrations, a "symmetric" successive boost application is Thomas gyration free, that is, it is equivalent to the application of a single boost. Three illustrative examples are given in the following three boost identities

$$
\begin{aligned}
B(\mathbf{u}) B(\mathbf{u})\left(\begin{array}{c}
t \\
\mathbf{w}
\end{array}\right) & =B(2 \otimes \mathbf{u})\left(\begin{array}{c}
t \\
\mathbf{w}
\end{array}\right) \\
B(\mathbf{v}) B(\mathbf{u}) B(\mathbf{v})\left(\begin{array}{c}
t \\
\mathbf{w}
\end{array}\right) & =B(\mathbf{v} \oplus(\mathbf{u} \oplus \mathbf{v}))\left(\begin{array}{c}
t \\
\mathbf{w}
\end{array}\right) \\
B(\mathbf{v}) B(\mathbf{u}) B(\mathbf{u}) B(\mathbf{v})\left(\begin{array}{c}
t \\
\mathbf{w}
\end{array}\right) & =B(\mathbf{v} \oplus(\mathbf{u} \oplus(\mathbf{u} \oplus \mathbf{v})))\left(\begin{array}{c}
t \\
\mathbf{w}
\end{array}\right) \\
& =B(2 \otimes(\mathbf{u} \oplus \mathbf{v}))\left(\begin{array}{c}
t \\
\mathbf{w}
\end{array}\right)
\end{aligned}
$$

where $2 \otimes \mathbf{u}=\mathbf{u} \oplus \mathbf{u}$ and, accordingly, $2 \otimes(\mathbf{u} \oplus \mathbf{v})=(\mathbf{u} \oplus \mathbf{v}) \oplus(\mathbf{u} \oplus \mathbf{v})$.

\section{The Abstract Lorentz Group}

We now extend the gyrocommutative gyrogroup of abstract Lorentz boosts to a group of abstract Lorentz transformations. Let $(G, \oplus)$ be any gyrocommutative gyrogroup (as, for instance, the Einstein gyrogroup $\left(\mathbb{V}_{c}, \oplus_{\mathrm{E}}\right)$ ), and let $\operatorname{Aut}_{0}(G, \oplus)$ be any subgroup of its automorphism group $\operatorname{Aut}(G, \oplus)$ that contains all the gyroautomorphisms of $(G, \oplus)$. Furthermore, let $(\mathbb{R} \times G, \oplus, \cdot, \rho)$ be the associated spacetime gyrocommutative gyrogroup equipped with a cocyclic map $\rho$. For any $V \in \operatorname{Aut}_{0}(G, \oplus)$ we use the notation

$$
\left(\begin{array}{c}
t \\
V \mathbf{v}
\end{array}\right)=E(V)\left(\begin{array}{c}
t \\
\mathbf{v}
\end{array}\right)
$$


$t \in \mathbb{R}, \mathbf{v} \in G$, so that, for instance,

$$
E(\operatorname{gyr}[\mathbf{u}, \mathbf{v}])=\operatorname{Gyr}[\mathbf{u}, \mathbf{v}]
$$

or, equivalently but in full notation

$$
E(\operatorname{gyr}[\mathbf{u}, \mathbf{v}])\left(\begin{array}{c}
t \\
\mathbf{w}
\end{array}\right)=\left(\begin{array}{c}
t \\
\operatorname{gyr}[\mathbf{u}, \mathbf{v}] \mathbf{w}
\end{array}\right)=\operatorname{Gyr}[\mathbf{u}, \mathbf{v}]\left(\begin{array}{c}
t \\
\mathbf{w}
\end{array}\right)
$$

as we see from (36) and (32).

The composition of an abstract boost and an automorphism gives an abstract Lorentz transformation. The abstract Lorentz transformation $L(\mathbf{v}, V)$ of the abstract spacetime $(\mathbb{R} \times G, \oplus, \cdot, \rho)$ is parameterized by a velocity parameter $\mathbf{v} \in G$ and an orientation parameter $V \in \operatorname{Aut}_{0}(G, \oplus)$. It is given by the equation

$$
L(\mathbf{v}, V)\left(\begin{array}{c}
t \\
\mathbf{w}
\end{array}\right)=B(\mathbf{v}) E(V)\left(\begin{array}{c}
t \\
\mathbf{w}
\end{array}\right)
$$

so that a Lorentz transformation is a boost preceded by an automorphism.

The set of all abstract Lorentz transformations of a given abstract spacetime forms a group with group operation given by transformation composition. Indeed, the abstract Lorentz transformation composition law is given by the equation

$$
L(\mathbf{u}, U) L(\mathbf{v}, V)=L(\mathbf{u} \oplus U \mathbf{v}, \operatorname{Gyr}[\mathbf{u}, U \mathbf{v}] U V)
$$

for all $\mathbf{u}, \mathbf{v} \in G$ and $U, V \in \operatorname{Aut}_{0}(G, \oplus)$, as verified in [26]. The Lorentz transformation composition law (40) is given by parameter composition called a $g y$ rosemidirect product. The latter, in turn, is fully analogous to the familiar semidirect product in group theory [14]. Hence,

i) the Lorentz group is a gyrosemidirect product group of a gyrocommutative gyrogroup of boosts and a group of automorphisms (which are 3-space rotations in the concrete realizations of interest in physics) just as

ii) the Galilei group is a semidirect product group of a commutative group of boosts and a group of automorphisms (that is, 3-space rotations).

Having the abstract Lorentz transformation group in hand, we can now realize it by concrete examples that result in various Lorentz groups. The abstract Lorentz boost comprises of

1) A set $G$ of objects called (relative) velocities that forms a gyrocommutative gyrogroup $(G, \oplus),(26)$. 
2a) A cocyclic map $\rho(\mathbf{v})$ that determines, and that is determined by, the invariant norm (30) of spacetime.

2b) An invariant norm of spacetime, $t / \rho(\mathbf{v}),(24)$, that determines a legitimate cocyclic map $\rho(\mathbf{v})$.

Hence, to single out a unique concrete example of a Lorentz transformation group when realizing the abstract Lorentz group, one must select

1) A concrete gyrocommutative gyrogroup $(G, \oplus)$ of objects called "velocities", and

2) A concrete invariant norm of spacetime that gives rise to a legitimate cocyclic map.

Accordingly, in Section 7 we realize the abstract Lorentz transformation group by selecting the Einstein gyrogroup of coordinate velocities and the standard relativistic norm, resulting in the standard (homogeneous, proper, orthochronous) Lorentz group. By recovering the standard Lorentz group as a concrete example of the abstract Lorentz group we demonstrate the ability of the abstract Lorentz group to generate concrete examples of various finite and infinite Lorentz groups, some of which could prove useful in physics.

In Section 9 we realize the abstract Lorentz transformation group by selecting the proper velocity gyrogroup and a norm given by the positively valued proper time. In Section 10 we demonstrate the realization of the abstract Lorentz group by a concrete Lorentz group that keeps invariant the four-dimensional Euclidean norm of spacetime. Finally, in Section 11 we present the Galilei transformation group as a concrete example of the abstract Lorentz group.

Remark 3. In group theory one starts from the notion of the abstract group which, in turn, is realized by various concrete examples. Clearly, the resulting concrete groups need not be isomorphic. In full analogy, the abstract Lorentz group that we define in this section can be realized by various concrete examples. But, also in full analogy with realizations of the abstract group, resulting concrete examples of Lorentz groups need not be experimentally equivalent.

Indeed, special care is taken in the realization of the abstract Lorentz group that we present in Section 9 in order to insure that it remains experimentally equivalent to the one in Section 7.

In contrast, the realization of the abstract Lorentz group that we present in Section 10 is experimentally distinguishable from the ones in Sections 7 and 9. 


\section{Realizing The Abstract Lorentz Group - Example I: The Standard, Coordinate-Time Lorentz Group}

To recover the standard (homogeneous, proper, orthochronous) Lorentz group from the abstract Lorentz group

1) we realize the abstract gyrocommutative gyrogroup $(G, \oplus)$ by Einstein gyrogroup $\left(\mathbb{R}_{c}^{3}, \oplus_{\mathrm{E}}\right)$, where Einstein addition $\oplus_{\mathrm{E}}$ in the Euclidean ball $\mathbb{R}_{c}^{3}$ is given by (4). Furthermore,

2) we realize the abstract spacetime norm (24) by the standard relativistic norm

$$
\left\|\left(\begin{array}{c}
t \\
\mathbf{v}
\end{array}\right)\right\|=\sqrt{t^{2}-\frac{\|\mathbf{x}\|^{2}}{c^{2}}}=\frac{t}{\gamma_{\mathbf{v}}}
$$

where $\mathbf{x}=\mathbf{v} t$ as in (2), and where $\gamma_{\mathbf{v}}$ is the Lorentz gamma factor (5). Rewriting (41) in space, rather than velocity, notation (2)-(3), it takes the familiar form

$$
\left\|\left(\begin{array}{l}
t \\
\mathbf{x}
\end{array}\right)\right\|=\sqrt{t^{2}-\frac{\|\mathbf{x}\|^{2}}{c^{2}}}=\frac{t}{\gamma_{\mathbf{v}}} .
$$

Comparing (41) with (24) we see that our choice of the spacetime norm (41) determines the cocyclic map $\rho(\mathbf{v})$

$$
\rho(\mathbf{v})=\gamma_{\mathbf{v}}=\frac{1}{\sqrt{1-\frac{\|\mathbf{v}\|^{2}}{c^{2}}}} .
$$

The resulting cocyclic map $\rho(\mathbf{v})$ in (43) is legitimate since it satisfies the conditions (19) and (20). To see that condition (19) is satisfied we note that the gyrations of the Einstein gyrocommutative gyrogroup $\left(\mathbb{R}_{c}^{3}, \oplus_{\mathrm{E}}\right)$ are rotations of $\mathbb{R}_{c}^{3}$ about its origin, and that $\rho(\mathbf{v})$ is clearly invariant under rotations.

i) Realizing the gyrocommutative gyrogroup binary operation $\oplus$ by Einstein velocity addition $\oplus_{\mathrm{E}}$ in step 1), and

ii) selecting a spacetime norm that realizes the abstract cocyclic map $\rho(\mathbf{v})$, $\mathbf{v} \in G$, by the concrete, legitimate cocyclic map $\gamma_{\mathbf{v}}, \mathbf{v} \in \mathbb{R}_{c}^{3}$, in step 2 ),

we can now realize the boost application to spacetime (28), obtaining

$$
B(\mathbf{u})\left(\begin{array}{c}
t \\
\mathbf{v}
\end{array}\right)=\left(\begin{array}{c}
\frac{\gamma_{\mathbf{u} \oplus_{\mathrm{E}} \mathbf{v}}}{\gamma_{\mathbf{v}}} t \\
\mathbf{u} \oplus_{\mathrm{E}} \mathbf{v}
\end{array}\right)=\left(\begin{array}{c}
\gamma_{\mathbf{u}}\left(1+\frac{\mathbf{u} \cdot \mathbf{v}}{c^{2}}\right) t \\
\mathbf{u} \oplus_{\mathrm{E}} \mathbf{v}
\end{array}\right)
$$


where we employ the gamma identity (6), and use the velocity notation (3).

Translating (44) from velocity into space notation of spacetime, (2) - (3), and noting that $\mathbf{x}=\mathbf{v} t$, we have

$$
\begin{aligned}
B(\mathbf{u})\left(\begin{array}{c}
t \\
\mathbf{x}
\end{array}\right) & =\left(\begin{array}{c}
\gamma_{\mathbf{u}}\left(t+\frac{1}{c^{2}} \mathbf{u} \cdot \mathbf{v} t\right) \\
\left(\mathbf{u} \oplus_{\mathrm{E}} \mathbf{v}\right) \gamma_{\mathbf{u}}\left(1+\frac{1}{c^{2}} \mathbf{u} \cdot \mathbf{v}\right) t
\end{array}\right) \\
& =\left(\begin{array}{c}
\gamma_{\mathbf{u}}\left(t+\frac{1}{c^{2}} \mathbf{u} \cdot \mathbf{x}\right) \\
\gamma_{\mathbf{u}} \mathbf{u} t+\mathbf{x}+\frac{1}{c^{2}} \frac{\gamma_{\mathbf{u}}^{2}}{1+\gamma_{\mathbf{u}}}(\mathbf{u} \cdot \mathbf{x}) \mathbf{u}
\end{array}\right)=\left(\begin{array}{c}
t^{\prime} \\
\mathbf{x}^{\prime}
\end{array}\right)
\end{aligned}
$$

The boost application in (45) is recognized in the literature [26] as the standard Lorentz boost of the special theory of relativity, that takes spacetime coordinates $(t, \mathbf{x})^{t}$ into spacetime coordinates $\left(t^{\prime}, \mathbf{x}^{\prime}\right)^{t}$, and that keeps the relativistic norm (42) invariant.

In order to extend the standard Lorentz boost to the standard Lorentz transformation we note that the group $\mathrm{SO}(3)$ of all rotations of the Euclidean ball $\mathbb{R}_{c}^{3}$ about its origin forms a subgroup of the automorphism group $\operatorname{Aut}\left(\mathbb{R}_{c}^{3}, \oplus_{\mathrm{E}}\right)$ that contains all the gyroautomorphisms of Einstein gyrogroup $\left(\mathbb{R}_{c}^{3}, \oplus_{\mathrm{E}}\right)$. We, accordingly, realize the abstract automorphism subgroup $\operatorname{Aut}_{0}(G, \oplus)$ by the concrete automorphism subgroup $\operatorname{Aut}_{0}\left(\mathbb{R}_{c}^{3}, \oplus_{\mathrm{E}}\right)=\mathrm{SO}(3)$. This realization of the abstract Lorentz boost and the abstract automorphism subgroup of the abstract Lorentz group gives the standard Lorentz group which, in space notation takes the form

$$
L(\mathbf{u}, U)\left(\begin{array}{c}
t \\
\mathbf{x}
\end{array}\right)=\left(\begin{array}{c}
\gamma_{\mathbf{u}}\left(t+\frac{1}{c^{2}} \mathbf{u} \cdot U \mathbf{x}\right) \\
\gamma_{\mathbf{u}} \mathbf{u} t+U \mathbf{x}+\frac{1}{c^{2}} \frac{\gamma_{\mathbf{u}}^{2}}{1+\gamma_{\mathbf{u}}}(\mathbf{u} \cdot U \mathbf{x}) \mathbf{u}
\end{array}\right)
$$

$\mathbf{u} \in \mathbb{R}_{c}^{3}, U \in \mathrm{SO}(3)$, and it keeps the relativistic norm (42)

$$
\left\|\left(\begin{array}{l}
t \\
\mathbf{x}
\end{array}\right)\right\|=\sqrt{t^{2}-\frac{\|\mathbf{x}\|^{2}}{c^{2}}}
$$

invariant. Here $\mathbf{x} \in \mathbb{R}_{c, t}^{3}$

$$
\mathbb{R}_{c, t}^{3}=\left\{\mathbf{x} \in \mathbb{R}^{3} ;\|\mathbf{x}\|<c t\right\}
$$

for all $t>0$.

Finally, being a realization of the abstract Lorentz transformation (39), the standard Lorentz transformation (46) possesses the group composition law (40)

$$
L(\mathbf{u}, U) L(\mathbf{v}, V)=L\left(\mathbf{u} \oplus_{\mathrm{E}} U \mathbf{v}, \operatorname{Gyr}[\mathbf{u}, U \mathbf{v}] U V\right)
$$


for all $\mathbf{u}, \mathbf{v} \in \mathbb{R}_{c}^{3}$ and $U, V \in \mathrm{SO}(3)$.

This composition law represents the standard Lorentz group as the gyrosemidirect product of

1) a gyrocommutative gyrogroup of boosts $B(\mathbf{v}), \mathbf{v} \in \mathbb{R}_{c}^{3}$, isomorphic to the Einsteinian gyrogroup $\left(\mathbb{R}_{c}^{3}, \oplus_{\mathrm{E}}\right)$ of relativistically admissible velocities, and

2) the group $\mathrm{SO}(3)$.

This representation is fully analogous to the familiar representation of the Galilean group as the semidirect product of

1) a commutative group of Galilean boosts, isomorphic to the Newtonian group $\left(\mathbb{R}^{3},+\right)$ of Newtonian velocities, and

2) the group $\mathrm{SO}(3)$.

The parameterization of the Lorentz group $L(\mathbf{v}, V)$ by a velocity, $\mathbf{v} \in \mathbb{R}_{c}^{3}$, and an orientation, $V \in \mathrm{SO}(3)$, parameters proves useful in the resulting Lorentz transformation composition law (49). It is a composition law given by parameter composition which, in turn, involves Einstein velocity addition. The interplay between Einsteinian relativity, based on Einstein addition of 3-velocities, and Minkowskian relativity, based on Lorentz transformation of 4-velocities, is clearly seen in the Lorentz transformation composition law (49). The approaches of Einstein and Minkowski to special relativity, thus, complement each other rather than compete with one another. The term "Minkowskian relativity" was coined by Pyenson in [18, p. 146]. The historical struggle between Einsteinian relativity and Minkowskian relativity is skillfully described by Walter in [29] where, for the first time, the term "Minkowskian relativity" appears in a title.

In the same way that we recovered the standard Lorentz group from the abstract one, other concrete Lorentz groups can be uncovered. An example of uncovering the proper-time Lorentz group is presented in Section 9 following the presentation of the proper velocity gyrogroup in Section 8 .

\section{The Proper Velocity Gyrogroup}

Coordinate time, or observer's time, is the time $t$ of a moving object measured by an observer at rest. Proper time, or traveller's time, is the time $\tau$ of a moving object measured by a co-moving observer. A detailed presentation of the proper time in a form that proves useful for the present article is found in [31, Section 6.2]. 
The coordinate time, $t$, and the proper time, $\tau$, of a uniformly moving object with relative velocity $\mathbf{v} \in \mathbb{R}_{c}^{3}$ measured by coordinate time, are related by the equation

$$
t=\gamma_{\mathbf{v}} \tau
$$

Accordingly, the relative velocities $\mathbf{v}$ and $\mathbf{w}$ of an object measured by its coordinate time and proper time, respectively, are related by the equations

$$
\mathbf{w}=\gamma_{\mathbf{v}} \mathbf{v} \in \mathbb{R}^{3}, \quad \mathbf{v}=\beta_{\mathbf{w}} \mathbf{w} \in \mathbb{R}_{c}^{3}
$$

where $\gamma_{\mathbf{v}}$ is the gamma factor (5) and where $\beta_{\mathbf{w}}$ is the beta factor given by the equation

$$
\beta_{\mathbf{w}}=\frac{1}{\sqrt{1+\frac{\|\mathbf{w}\|^{2}}{c^{2}}}} .
$$

Gill and Zachary [9] attribute the relations in (51) to G. Schott.

Let $\phi: \mathbb{R}^{3} \rightarrow \mathbb{R}_{c}^{3}$ be the bijective map that (51) suggests

$$
\phi \mathbf{w}=\beta_{\mathbf{w}} \mathbf{w}=\mathbf{v}
$$

with inverse $\phi^{-1}: \mathbb{R}_{c}^{3} \rightarrow \mathbb{R}^{3}$

$$
\phi^{-1} \mathbf{v}=\gamma_{\mathbf{v}} \mathbf{v}=\mathbf{w} .
$$

Then Einstein addition $\oplus_{\mathrm{E}}$ in $\mathbb{R}_{\mathrm{C}}^{3}$ induces the binary operation $\oplus_{\mathrm{U}}$ in $\mathbb{R}^{3}$

$$
\mathbf{w}_{1} \oplus_{\mathrm{U}} \mathbf{w}_{2}=\phi^{-1}\left(\phi \mathbf{w}_{1} \oplus_{\mathrm{E}} \phi \mathbf{w}_{2}\right)
$$

$\mathbf{w}_{1}, \mathbf{w}_{2} \in \mathbb{R}^{3}$, thus uncovering the proper velocity composition law in $\mathbb{R}^{3}$. Using software for symbolic manipulation, it can be shown that the binary operation $\oplus_{\mathrm{U}}$, (55), in $\mathbb{R}^{3}$ is given by the equation

$$
\mathbf{u} \oplus_{\mathrm{U}} \mathbf{v}=\mathbf{u}+\mathbf{v}+\left\{\frac{\beta_{\mathbf{u}}}{1+\beta_{\mathbf{u}}} \frac{\mathbf{u} \cdot \mathbf{v}}{c^{2}}+\frac{1-\beta_{\mathbf{v}}}{\beta_{\mathbf{v}}}\right\} \mathbf{u}
$$

where $\beta_{\mathbf{v}}$ is the beta factor, satisfying the beta identity

$$
\beta_{\mathbf{u} \oplus_{\mathrm{U}} \mathbf{v}}=\frac{\beta_{\mathbf{u}} \beta_{\mathbf{v}}}{1+\beta_{\mathbf{u}} \beta_{\mathbf{v}} \frac{\mathbf{u} \cdot \mathbf{v}}{c^{2}}}
$$

for all $\mathbf{u}, \mathbf{v} \in \mathbb{R}^{3}$.

Owing to the isomorphism $\phi$, the groupoid $\left(\mathbb{R}^{3}, \oplus_{\mathrm{U}}\right)$ forms a gyrocommutative gyrogroup of proper velocities, isomorphic to Einstein gyrogroup $\left(\mathbb{R}_{c}^{3}, \oplus_{\mathrm{E}}\right)$ of coordinate velocities. The proper velocity gyrogroup $\left(\mathbb{R}^{3}, \oplus_{U}\right)$ is also known as an Ungar gyrogroup, a term coined by Chen in [1]. Having the proper velocity gyrogroup in hand, we are now in a position to realize the abstract Lorentz transformation group by a proper-time Lorentz group. 


\section{Realizing The Abstract Lorentz Group - Example II: The Proper-Time Lorentz Group}

To uncover the proper-time Lorentz group from the abstract Lorentz group

1) we realize the abstract gyrocommutative gyrogroup $(G, \oplus)$ by the propervelocity gyrogroup $\left(\mathbb{R}^{3}, \oplus_{\mathrm{U}}\right)$, where the proper velocity addition $\oplus_{\mathrm{U}}$ in the Euclidean 3 -space $\mathbb{R}^{3}$ is given by (56). Furthermore,

2) we realize the abstract spacetime norm (24) by the positive valued proper time $\tau$

$$
\left\|\left(\begin{array}{l}
\tau \\
\mathbf{v}
\end{array}\right)\right\|=\tau
$$

$\mathbf{v} \in \mathbb{R}^{3}, \tau>0$, since we seek a proper-time Lorentz transformation that keeps the proper time invariant. Selecting any different norm would break the convention made in step 1) to replace coordinate time by proper time. Breaking the convention, as we will do in Section 10, results in a new Lorentz group which is experimentally distinguishable from the standard one. Rewriting (58) in space, rather than velocity, notation (2) - (3), it takes the form

$$
\left\|\left(\begin{array}{l}
\tau \\
\mathbf{x}
\end{array}\right)\right\|=\tau
$$

$\mathbf{x}=\mathbf{v} \tau \in \mathbb{R}^{3}, \tau>0$.

Comparing (58) with (24) we see that our choice of the spacetime norm (58) determines the cocyclic map

$$
\rho(\mathbf{v})=1
$$

for all $\mathbf{v} \in \mathbb{R}^{3}$. The resulting trivial cocyclic map is legitimate since it, trivially, satisfies the conditions (19) and (20).

i) Realizing the gyrocommutative gyrogroup binary operation $\oplus$ by the proper velocity addition $\oplus_{\mathrm{U}}$ in step 1$)$, and

ii) selecting a spacetime norm that realizes the abstract cocyclic map $\rho(\mathbf{v})$, $\mathbf{v} \in G$, by the concrete, legitimate cocyclic map $\rho(\mathbf{v})=1, \mathbf{v} \in \mathbb{R}^{3}$, in step 2), we can now realize the boost application to spacetime, (28), obtaining

$$
B_{p}(\mathbf{u})\left(\begin{array}{l}
\tau \\
\mathbf{v}
\end{array}\right)=\left(\begin{array}{c}
\tau \\
\mathbf{u} \oplus_{\mathrm{U}} \mathbf{v}
\end{array}\right) .
$$


Translating (61) from velocity to space notation of spacetime, (2) - (3), and noting that $\mathbf{x}=\mathbf{v} \tau$, we have

$$
\begin{aligned}
B_{p}(\mathbf{u})\left(\begin{array}{l}
\tau \\
\mathbf{x}
\end{array}\right) & =\left(\begin{array}{c}
\tau \\
\left(\mathbf{u} \oplus_{\mathrm{U}} \mathbf{v}\right) \tau
\end{array}\right)=\left(\begin{array}{c}
\tau \\
(\mathbf{u}+\mathbf{v}) \tau+\left(\frac{\beta_{\mathbf{u}}}{1+\beta_{\mathbf{u}}} \frac{\mathbf{u} \cdot \mathbf{v} \tau}{c^{2}}+\frac{1-\beta_{\mathbf{v}}}{\beta_{\mathbf{v}}} \tau\right) \mathbf{u}
\end{array}\right) \\
& =\left(\begin{array}{c}
\tau \\
\mathbf{x}+\frac{1}{1+\sqrt{1+\mathbf{u}^{2} / c^{2}}} \frac{\mathbf{u} \cdot \mathbf{x}}{c^{2}} \mathbf{u}+\sqrt{1+\mathbf{v}^{2} / c^{2}} \mathbf{u} \tau
\end{array}\right) \\
& =\left(\begin{array}{c}
1 \\
\mathbf{x}+\frac{1}{1+\sqrt{1+\mathbf{u}^{2} / c^{2}}} \frac{\mathbf{u} \cdot \mathbf{x}}{c^{2}} \mathbf{u}+\sqrt{\tau^{2}+\|\mathbf{x}\|^{2} / c^{2}} \mathbf{u}
\end{array}\right)=\left(\begin{array}{l}
\tau^{\prime} \\
\mathbf{x}^{\prime}
\end{array}\right)
\end{aligned}
$$

which is the proper-time Lorentz boost of the special theory of relativity, that takes spacetime coordinates $(\tau, \mathbf{x})^{t}$ into spacetime coordinates $\left(\tau^{\prime}, \mathbf{x}^{\prime}\right)^{t}$. Unlike the standard, coordinate-time Lorentz boost, the proper-time Lorentz boost is nonlinear.

In order to extend the proper-time Lorentz boost to the proper-time Lorentz transformation we note that the group $\mathrm{SO}(3)$ of all rotations of the Euclidean 3-space $\mathbb{R}^{3}$ about its origin forms a subgroup of the automorphism group $\operatorname{Aut}\left(\mathbb{R}^{3}, \oplus_{\mathrm{U}}\right)$ that contains all the gyroautomorphisms of the proper velocity gyrogroup $\left(\mathbb{R}^{3}, \oplus_{U}\right)$. We, accordingly, realize the abstract automorphism subgroup $\operatorname{Aut}_{0}(G, \oplus)$ by the 3 -space rotation group $\operatorname{Aut}_{0}\left(\mathbb{R}^{3}, \oplus_{\mathrm{U}}\right)=\mathrm{SO}(3)$. This realization of the abstract Lorentz boost and the abstract automorphism subgroup of the abstract Lorentz group gives the proper-time Lorentz group which, in space notation takes the form

$$
\begin{aligned}
L_{p}(\mathbf{u}, U)\left(\begin{array}{l}
\tau \\
\mathbf{x}
\end{array}\right) & =B_{p}(\mathbf{u}) E(U)\left(\begin{array}{l}
\tau \\
\mathbf{x}
\end{array}\right)=\left(\begin{array}{c}
\tau \\
\left(\mathbf{u} \oplus_{\mathrm{U}} U \mathbf{v}\right) \tau
\end{array}\right) \\
& =\left(\begin{array}{c}
\tau \\
U \mathbf{x}+\frac{1}{1+\sqrt{1+\mathbf{u}^{2} / c^{2}}} \frac{\mathbf{u} \cdot U \mathbf{x}}{c^{2}} \mathbf{u}+\sqrt{\tau^{2}+\|\mathbf{x}\|^{2} / c^{2}} \mathbf{u}
\end{array}\right)^{(63)}
\end{aligned}
$$

$\mathbf{u}, \mathbf{x} \in \mathbb{R}^{3}, U \in \mathrm{SO}(3), \tau>0$.

Finally, being a realization of the abstract Lorentz transformation (39), the propertime Lorentz transformation (63) possesses the group composition law (40)

$$
L_{p}(\mathbf{u}, U) L_{p}(\mathbf{v}, V)=L_{p}\left(\mathbf{u} \oplus_{\mathrm{E}} U \mathbf{v}, \operatorname{Gyr}[\mathbf{u}, U \mathbf{v}] U V\right)
$$


for all $\mathbf{u}, \mathbf{v} \in \mathbb{R}^{3}$ and $U, V \in \mathrm{SO}(3)$.

This composition law represents the proper-time Lorentz group as the gyrosemidirect product of

1) a gyrogroup of boosts $B_{p}(\mathbf{v}), \mathbf{v} \in \mathbb{R}^{3}$, isomorphic to the proper velocity gyrogroup $\left(\mathbb{R}^{3}, \oplus_{\mathrm{U}}\right)$ of relativistic proper velocities, and

2) the group $\mathrm{SO}(3)$.

\section{Realizing The Abstract Lorentz Group - Example III: A Lorentz Group With Euclidean Spacetime Norm}

We now present a third example of realizing the abstract Lorentz group. Following Montanus [16,17] we use the proper-time $\tau$ rather than coordinate time $t$ and select the spacetime invariant of the Lorentz group that we seek to be the four dimensional Euclidean metric

$$
\sqrt{\tau^{2}+\|\mathbf{x}\|^{2} / c^{2}}
$$

$\mathbf{x} \in \mathbb{R}^{3}, \tau>0$.

Selecting $\tau$, rather than (65), as an invariant of the proper-time Lorentz group that we seek is a matter of experimental fact that results from the convention of replacing coordinate time by proper time. This matter of experimental fact is compatible with the standard, coordinate-time Lorentz group.

Similarly, Montanus' selection of the four dimensional Euclidean metric (65) along with his selection of proper time rather than coordinate time is a matter of experimental fact rather than convention. This matter of experimental fact, however, is not compatible with the standard Lorentz group, as Gersten notes [7]. Hence, the Lorentz group that we uncover in this section as a realization of the abstract Lorentz group is experimentally distinguishable from the standard Lorentz group.

As in Section 9, we select the proper-velocity gyrogroup $\left(\mathbb{R}^{3}, \oplus_{\mathrm{U}}\right)$ as our realization of the abstract gyrocommutative gyrogroup $(G, \oplus)$. Conforming with the standard Lorentz group would force us to construct a Lorentz group that keeps the proper time invariant, as we did in Section 9. In this section, however, we select a different norm (65), to obtain a nonlinear Lorentz transformation group that is experimentally distinguishable from the standard Lorentz group.

To uncover this nonstandard Lorentz group from the abstract Lorentz group 
1) we realize the abstract gyrocommutative gyrogroup $(G, \oplus)$ by the propervelocity gyrogroup $\left(\mathbb{R}^{3}, \oplus_{\mathrm{U}}\right)$, where proper velocity addition $\oplus_{\mathrm{U}}$ in the Euclidean 3 -space $\mathbb{R}^{3}$ is given by (56). Furthermore,

2) we realize the abstract spacetime norm (24) by

$$
\left\|\left(\begin{array}{l}
\tau \\
\mathbf{v}
\end{array}\right)\right\|=\frac{\tau}{\beta_{\mathbf{v}}}
$$

$\mathbf{v} \in \mathbb{R}^{3}, \tau>0$, where $\beta_{\mathbf{v}}$ is the beta factor. Rewriting (66) in space, rather than velocity, notation, it takes the form

$$
\left\|\left(\begin{array}{l}
\tau \\
\mathbf{x}
\end{array}\right)\right\|=\frac{\tau}{\beta_{\mathbf{v}}}=\sqrt{\tau^{2}+\frac{\|\mathbf{x}\|^{2}}{c^{2}}}
$$

$\mathbf{x}=\mathbf{v} \tau \in \mathbb{R}^{3}, \tau>0$, thus resulting in the standard four-dimensional Euclidean norm for spacetime.

Comparing (66) with (24) we see that our choice of the spacetime norm (66) determines the cocyclic map

$$
\rho(\mathbf{v})=\beta_{\mathbf{v}}
$$

for all $\mathbf{v} \in \mathbb{R}^{3}$. The resulting cocyclic map is legitimate since it satisfies the conditions (19) and (20).

i) Realizing the gyrocommutative gyrogroup binary operation $\oplus$ by the proper velocity addition $\oplus_{\mathrm{U}}$ in step 1 ), and

ii) selecting a spacetime norm that realizes the abstract cocyclic map $\rho(\mathbf{v}), \mathbf{v} \in$ $G$, by the concrete, legitimate cocyclic map $\rho(\mathbf{v})=\beta_{\mathbf{v}}, \mathbf{v} \in \mathbb{R}^{3}$, in step 2), we can now realize the boost application to spacetime, $(28)$, obtaining

$$
B_{s}(\mathbf{u})\left(\begin{array}{c}
\tau \\
\mathbf{v}
\end{array}\right)=\left(\begin{array}{c}
\frac{\beta_{\mathbf{u} \oplus_{\mathrm{U}} \mathbf{v}} \tau}{\beta_{\mathbf{v}}} \\
\mathbf{u} \oplus_{\mathrm{U}} \mathbf{v}
\end{array}\right)=\left(\begin{array}{c}
\frac{\beta_{\mathbf{u}}}{1+\beta_{\mathbf{u}} \beta_{\mathbf{v}} \mathbf{u} \cdot \mathbf{v} / c^{2}} \tau \\
\mathbf{u} \oplus_{\mathrm{U}} \mathbf{v}
\end{array}\right)
$$

where we employ the beta identity (57).

Translating (69) from velocity to space notation of spacetime, (2) - (3), and noting that $\mathbf{x}=\mathbf{v} \tau$, we can find that while the semi-Euclidean Lorentz boost keeps the Euclidean norm (67) invariant, it is too complicated. Hence, the passage from the relativistic norm (42) to the Euclidean norm (67) results in a complicated nonlinear boost that is experimentally distinguishable from the standard Lorentz boost. 


\section{Realizing The Abstract Lorentz Group - Example IV: The Galilean Group}

We now present a fourth example of realizing the abstract Lorentz group in which we use the coordinate time, $t$, and select the spacetime invariant of the "Lorentz group" that we seek to be $t$, as in Galilean relativity. Furthermore, we select the velocity "gyrogroup" $\left(\mathbb{R}^{3},+\right)$ as our realization of the abstract gyrocommutative gyrogroup $(G, \oplus)$, where + is the ordinary vector addition in $\mathbb{R}^{3}$. Clearly, the gyrogroup $\left(\mathbb{R}^{3},+\right)$ is, in fact, a group and, accordingly, all its gyrations are trivial. To uncover the resulting concrete example of a "Lorentz group"

1) we realize the abstract gyrocommutative gyrogroup $(G, \oplus)$ by the Newtonian velocity group $\left(\mathbb{R}^{3},+\right)$ (we may note that a group is a special gyrogroup all the gyrations of which are trivial); and

2) we realize the abstract spacetime norm (24) by

$$
\left\|\left(\begin{array}{c}
t \\
\mathbf{v}
\end{array}\right)\right\|=t
$$

$\mathbf{v} \in \mathbb{R}^{3}, t \in \mathbb{R}^{3}$. Rewriting (70) in space, rather than velocity, notation (2) - (3), it takes the form

$$
\left\|\left(\begin{array}{c}
t \\
\mathbf{x}
\end{array}\right)\right\|=t
$$

$\mathbf{x}=\mathbf{v} t \in \mathbb{R}^{3}, t \in \mathbb{R}^{3}$.

Comparing (70) with (24) we see that our choice of the spacetime norm (70) determines the cocyclic map

$$
\rho(\mathbf{v})=1
$$

for all $\mathbf{v} \in \mathbb{R}^{3}$. The resulting trivial cocyclic map is legitimate since it trivially satisfies the conditions (19) and (20).

i) Realizing the gyrocommutative gyrogroup binary operation $\oplus$ by the ordinary vector addition + in step 1 ), and

ii) selecting a spacetime norm that realizes the abstract cocyclic map $\rho(\mathbf{v})$, $\mathbf{v} \in G$, by the concrete, legitimate cocyclic map $\rho(\mathbf{v})=1, \mathbf{v} \in \mathbb{R}^{3}$, in step 2), 
we can now realize the boost application to spacetime, (28), obtaining

$$
B_{g}(\mathbf{u})\left(\begin{array}{c}
t \\
\mathbf{v}
\end{array}\right)=\left(\begin{array}{c}
\frac{\rho_{\mathbf{u}+\mathbf{v}}}{\rho_{\mathbf{v}}} \\
\mathbf{u}+\mathbf{v}
\end{array}\right)=\left(\begin{array}{c}
t \\
\mathbf{u}+\mathbf{v}
\end{array}\right)
$$

Translating (73) from velocity to space notation of spacetime, (2) - (3), and noting that $\mathbf{x}=\mathbf{v} t$, we have

$$
B_{g}(\mathbf{u})\left(\begin{array}{c}
t \\
\mathbf{x}
\end{array}\right)=\left(\begin{array}{c}
t \\
(\mathbf{u}+\mathbf{v}) t
\end{array}\right)=\left(\begin{array}{c}
t \\
\mathbf{x}+\mathbf{u} t
\end{array}\right)
$$

thus recovering the Galilei boost of Galilean relativity as a concrete example of the abstract Lorentz group.

\section{Conclusion}

Employing either coordinate time or proper time in relativity physics is a matter of convention. Proper time is an important parameter in relativity physics. Yet, the corresponding proper-time Lorentz transformation that are found in the literature is experimentally not equivalent to the standard, coordinate-time Lorentz transformation group and, therefore, must be erroneous.

In order to unambiguously determine the proper-time Lorentz transformation group, which must remain experimentally equivalent to the standard Lorentz group, one must understand the algebraic structure of the Lorentz group. Once understood, one can translate the standard Lorentz group into the non-standard, but experimentally equivalent, proper-time Lorentz group, as well as into other Lorentz groups that need not be experimentally equivalent to the standard one.

Having the standard (homogeneous, proper, orthochronous) Lorentz group in hand, one is able to generate concrete Lorentz groups by realizing the abstract one in various ways. The abstract Lorentz transformation group is presented in Section 6 , following which four examples of concrete realizations of the abstract Lorentz transformation group are presented in Sections $7-11$.

1) In Section 7 the standard Lorentz group is recovered as a concrete example of the abstract Lorentz group.

2) In Section 9 the proper-time Lorentz transformation group is uncovered as a concrete example of the abstract Lorentz group. 
3) In Section 10 a Lorentz group that keeps invariant a four-dimensional Euclidean norm of spacetime is constructed. As expected, it is not experimentally equivalent to the standard Lorentz group.

4) In section 11 the Galilean transformation group is recovered as a concrete example of the abstract Lorentz group.

The goal of this paper is to present the abstract Lorentz group and to uncover the proper-time Lorentz group, Section 9, as a concrete realization. The other examples of concrete realizations of the abstract Lorentz group in Sections 7, 10, and 11 are presented only in order to demonstrate the use of the abstract Lorentz group for the construction of various concrete Lorentz groups.

The power and elegance of gyrogroup theoretic techniques for uncovering the proper-time Lorentz group have thus been demonstrated. This extraordinary power and elegance is not limited to gyrogroups. Some gyrocommutative gyrogroups admit scalar product, giving rise to gyrovector spaces. The latter, in turn, are useful in analytic hyperbolic geometry [28] and its applications in special relativity theory [26]. Further extension gives rise to Lie gyrovector spaces, studied by Kasparian and Ungar in [12].

\section{References}

[1] Chen J. and Ungar A., From the Group $S L(2, \mathbb{C})$ to Gyrogroups and Gyrovector Spaces and Hyperbolic Geometry, Found. Phys. 31 (2001) 16111639.

[2] Einstein A., Zur Elektrodynamik Bewegter Körper. Ann. Physik (Leipzig) 17 (1905) 891-921.

[3] Einstein A., Einstein's Miraculous Years: Five Papers that Changed the Face of Physics. Princeton, Princeton, NJ, 1998. Edited and introduced by J. Stachel. Includes bibliographical references and Einstein's dissertation on the determination of molecular dimensions - Einstein on Brownian motion - Einstein on the theory of relativity - Einstein's early work on the quantum hypothesis. A new English translation of Einstein's 1905 paper on pp. 123160.

[4] Foguel T. and Ungar A., Involutory Decomposition of Groups into Twisted Subgroups and Subgroups, J. Group Theory 3 (2000) 27-46.

[5] Foguel T. and Ungar A., Gyrogroups and the Decomposition of Groups into Twisted Subgroups and Subgroups, Pac. J. Math 197 (2001) 1-11. 
[6] Frisch D. and Smith J., Measurement of the Relativistic Time Dilation Using $\mu$-mesons, Amer. J. Phys. 31 (1963) 342-355.

[7] Gersten A., Euclidean Special Relativity, In Proceedings, Part 1, IARD 2002 (Washington, DC) 33 (2003) 1237-1251.

[8] Gill T. and Zachary W., Proper-Time Formulation of Classical Electrodynamics, J. Nonlinear Math. Phys. 4 (1997) 418-425.

[9] Gill T., Zachary W. and Lindesay J., The Classical Electron, Found. Phys. 31 (2001) 1299-1355.

[10] Hall M. and Anderson M., Invariant Hamiltonians for Relativistic Particles, Amer. J. Phys. 63 (1995) 633-637.

[11] Hlavatý V., Proper Time, Apparent Time, and Formal Time in the Twin Paradox, J. Math. Mech. 9 (1960) 733-744.

[12] Kasparian A. and Ungar A., Lie Gyrovector Spaces, J. Geom. Symm. Phys. 1 (2004) 3-53.

[13] Kiechle H., Theory of K-loops, Springer, Berlin, 2002.

[14] Kinyon M. and Jones O., Loops and Semidirect Products, Comm. Algebra 28 (2000) 4137-4164.

[15] Lindesay J. and Gill T., Canonical Proper Time Formulation for Physical Systems, Found. Phys. 34 (2004) 169-182.

[16] Montanus J., Proper Time Physics, Hadronic J. 22 (1999) 625-673.

[17] Montanus J., Proper-Time Formulation of Relativistic Dynamics, In: Proceedings IARD 2000, Part 4 (Ramat Gan, 2000) 31 (2001) pp. 1357-1400.

[18] Pyenson L., Relativity in Late Wilhelmian Germany: The Appeal to a Preestablished Harmony Between Mathematics and Physics, Arch. Hist. Exact Sci. 27 (1982) 137-155.

[19] Rózga K., On Central Extensions of Gyrocommutative Gyrogroups, Pacific J. Math. 193 (2000) 201-218.

[20] Sexl R. and Urbantke H., Relativity, Groups, Particles, Springer, Vienna, 2001. Special Relativity and Relativistic Symmetry in Field and Particle Physics, Revised and translated from the third German (1992) edition by H. Urbantke.

[21] Silberstein L., The Theory of Relativity, MacMillan, London, 1914.

[22] Ungar A., Thomas Rotation and the Parametrization of the Lorentz Transformation Group, Found. Phys. Lett. 1 (1988) 57-89.

[23] Ungar A., The Relativistic Noncommutative Nonassociative Group of Velocities and the Thomas Rotation, Resultate Math. 16 (1989) 168-179. 
[24] Ungar A., Thomas Precession and its Associated Grouplike Structure, Amer. J. Phys. 59 (1991) 824-834.

[25] Ungar A., Thomas Precession: Its Underlying Gyrogroup Axioms and their Use in Hyperbolic Geometry and Relativistic Physics, Found. Phys. 27 (1997) 881-951.

[26] Ungar A., Beyond the Einstein Addition Law and its Gyroscopic Thomas Precession: The Theory of Gyrogroups and Gyrovector Spaces, volume 117 of Fundamental Theories of Physics, Kluwer, Dordrecht, 2001.

[27] Ungar A., Einstein's Special Relativity: Unleashing the Power of its Hyperbolic Geometry, Comput. Math. Appl. 49 (2005) 187-221.

[28] Ungar A., Analytic Hyperbolic Geometry: Mathematical Foundations and Applications, World Scientific, Singapore, 2005.

[29] Walter S., The Non-Euclidean Style of Minkowskian Relativity, In: The Symbolic Universe, J. Gray (Ed.), Oxford Univ. Press, New York (1999) 91-127.

[30] Wegener M., A Classical Alternative to Special Relativity, Phys. Essays 8 (1995) 427-433.

[31] Woodhouse N., Special Relativity. Springer Undergraduate Mathematics Series, Springer, London, 2003.

[32] Yamaleev R. and Fernandez A., Proper-time Relativistic Dynamics on Hyperboloid, Found. Phys. Lett. 14 (2001) 323-339.

Abraham A. Ungar

Department of Mathematics

North Dakota State University

Fargo, North Dakota 58105

USA

E-mail: Abraham. Ungar@ndsu.edu 